\title{
COEXISTENCE SIMULATION OF DVB-T/T2 AND LTE800 IN SEAMCAT
}

\author{
Lukáš SENDREI*, Marián FELIX**, Juraj ORAVEC***, Stanislav MARCHEVSKÝ* \\ * Department of Electronics and Multimedia Communications, Faculty of Informatics and Electrical Engineering, \\ Technical University of Košice, Slovakia (e-mail: \{lukas.sendrei, stanislav.marchevsky\}@tuke.sk) \\ ** The Research Institute of Posts and Telecommunications, Banská Bystrica, Slovakia (e-mail: \{felix, joravec\}@vus.s)
}

\begin{abstract}
The development of modern, effective and intelligent wireless communication systems, which enable to provide multimedia services in high quality takes place more faster than in years before. The small frequency bandwidth gap of the $4 G$ systems represented by Long Term Evolution (LTE) and digital broadcast system Digital Video Broadcasting - Terrestrial and the second generation denoted as $T 2$ (DVB-T/T2) is responsible for the presence of different interference scenarios. This paper highlights the analytical background of both systems and the issue of coexistence. Moreover, computer simulations are used to evaluate the deployment of filters on the digital television receiver side as well as spectral emission mask on LTE base station in order to mitigate interference.
\end{abstract}

Keywords: LTE800, DVB-T/T2, coexistence

\section{INTRODUCTION}

The usage of wireless mobile networks is nowadays spreading in everyday like of most people and one can barely imagine the live without these services. Therefore, the ever increasing demand on stable wireless communication and multimedia services is a key factor for today's business. The decision to allocate the frequency band in range $790-862 \mathrm{MHz}$, in order to meet the requirements on mobile delivery of high quality video, audio, data transmission and many others, was made on the conference WRC2007 (World Radio Conference) [1]. However, this band is separated from the frequency band allocated for digital television broadcasting just by an $1 \mathrm{MHz}$ guard band. So it is obvious that there will be a large potential of interference with the received TV signals. We can assume that Channel 60 is the most vulnerable one [2].

At the beginning of the paper, we introduce the topic of DVB-T/T2 and LTE800, respectively, and the issue of its mutual interference. The main part is dedicated to the creation of interference scenarios between LTE800 and DVBT/T2 systems followed by computer simulations. The parameters of both systems are set in the way that they reflect the real world. We have chosen the broadcasting in the town Lučenec as a benchmark, because because of the high probability of the interference on Ch60. The simulations analyse the deployment of spectral mask on LTE transmitter, filter on the digital television receiver or their combination in order to reduce the interference risk. At the end, the simulation results are evaluated.

\section{DVB-T/T2}

\subsection{DVB-T}

DVB-T standard was set in 1997 and one year later was put into use for the transmission of digital terrestrial television [3]. In contrast to analog the principle of digital broadcasting is based on the transmission of digital high speed data stream. However, this streams are not well suited to transmission. Therefore, the data stream is first compressed via some type of video codec system (e.g.: MPEG2, MPEG-4 AVC/H.264) which removes redundant compo- nents from the pictures. This enables far more better utilizations of the frequency spectrum what practically means that in one channel we can integrate more video, audio and other data services instead of single television service and thus create the so-called multiplex.

DVB-T uses OFDM (Orthogonal Frequency Division Multiplexing) which splits the digital data stream into a large number of low speed digital streams, each of which digitally modulates and sets on a closely spaced adjacent carrier frequencies. There are two options for the number of carriers known as $2 \mathrm{k}$-mode (uses 1705 subcarriers) or 8k-mode (uses 6817 subcarriers) for DVB-T and offers 16QAM, 64-QAM, QPSK modulation constalations. DVB$\mathrm{T}$ has been adopted for digital television broadcasting in many countries, using mainly VHF $7 \mathrm{MHz}$ and UHF 8 $\mathrm{MHz}$ channels. The first generation is primarily used for transmission of the standard definition (SD) digital television broadcasting, but also high definition (HD) services are distributed. In general both . MPEG-2 and MPEG-4 AVC/H.264 source coding are being used. However. providing terrestrial HDTV services in DVB-T broadcasting is not an effective way.

\subsection{DVB-T2}

To meet the expectations of television users and broadcasters, the television transmission systems must adapt to new service requirements. The research of transmission technology has continued and new version for errorprotecting broadcast streams and also new types of modulating have been developed since the DVB-T standard was published. The last $2^{\text {nd }}$ generation standard for the terrestrial broadcasting is DVB-T2, which offers significant benefits compared to DVB-T [4]. This upgrade enables new high-quality services, such as the HDTV and UHDTV through terrestrial networks. It is characterised by higher spectral efficiency, which means that the comparable number of programmes with higher video and audio quality (HDTV, UHDTV) or larger number of programmes can be broadcasted. Also new advanced system of source coding MPEG-H/H.265 (also called HEVC) brings remarkable benefits. DVB-T2 uses up to 256-QAM modulation, plus 
higher number of carriers to provide higher bit rates. DVBT2 uses LDPC (Low Density Parity Check) and also BCH (Bose-Chaudhuri-Hocquengham) coding for error protection. There is also a new technique, known as Rotated Constellations, which has been introduced to provide additional robustness in certain conditions. DVB-T2 standard takes also care of transmitter equipments. Particularly in $32 \mathrm{k}$ mode, high power peaks are generated and thus the amplifier efficiency is decreased. A special feature called PAPR (Peak Average Power Ratio) reduction has been in included in the standard specification to limit these power peaks without losing information.

IF we want to compare DVB-T and DVB-T2 we need to take into account the following features:

- New generation of FEC (Forward Error Correction) and higher modulation constellation (256-QAM) which increases the capacity approximately by $30 \%$ and is close to the Shannon limit.

- The number of subcarriers in OFDM increased from $8 \mathrm{k}$ to $32 \mathrm{k}$.

- Changes in pilot symbol placement.

These improvements brought markable decrease of overhead costs in hand with better protections against errors which raises the capacity by $50 \%$ in comparison to DVB-T. DVB-T2 extended the possible channel capacity choices to 1.7 MHz, $5 \mathrm{MHz}$ and $10 \mathrm{MHz}$. The DVB-T2 system contains a few new properties to improve the reception in bad reception conditions:

- Rotated modulation constellation diagram (increased symbol receiving at higher symbol rates)

- PAPR reduction techniques (increased amplifier efficiency)

More detailed comparison between DVB-T and DVB-T2 can be found in Tab.1.

\section{LTE}

LTE is the newest wireless standard evolved from UMTS (Universal Mobile Telecommunications system). Its specifications were published by 3GPP (3th Generation Partnership Project) in the Release 8 in 2008. The system is purely packet based and uses IP, precisely MIP (Mobile IP). LTE has several advantages like low latency on the radio interface $(<10 \mathrm{~ms})$, spectral efficiency $(3-4 \times$ higher than in HSPA) and of course higher data rates up to $100 \mathrm{Mbit} / \mathrm{s}$. The LTE system is able to transmit high data rates, theoretically up to $100 \mathrm{Mbit} / \mathrm{s}$ at downlink and $50 \mathrm{Mbit} / \mathrm{s}$ at uplink. It offers also variable channel bandwidth from 1.4 up to $20 \mathrm{MHz}$. In contrast to earlier mobile system UMTS, LTE uses OFDM. LTE uses FDD and TDD as well as multiple antenna systems (MIMO).
Table 1 Parameter comparison between DVB-T and DVB-T2

\begin{tabular}{|c|c|c|}
\hline & DVB-T & DVB-T2 \\
\hline \multirow[t]{3}{*}{ FEC } & Convolution code + & $\mathrm{LDPC}+\mathrm{BHC}$ \\
\hline & Reed Solomon & $1 / 2,3 / 5,2 / 3$ \\
\hline & $1 / 2,2 / 3,3 / 4,5 / 6,7 / 8$ & $3 / 4,4 / 5,5 / 6$ \\
\hline \multirow[t]{4}{*}{ Modulation } & QPSK & QPSK \\
\hline & 16-QAM & 16-QAM \\
\hline & 64-QAM & 64-QAM \\
\hline & & 256-QAM \\
\hline \multirow[t]{4}{*}{ Guard interval } & $1 / 4,1 / 8$ & $1 / 4,19 / 256$ \\
\hline & $1 / 16,1 / 32$ & $1 / 8,19 / 128$ \\
\hline & & $1 / 16,1 / 32$ \\
\hline & & $1 / 128$ \\
\hline Number of & $2 \mathrm{k}, 8 \mathrm{k}$ & $1 \mathrm{k}, 2 \mathrm{k}, 4 \mathrm{k}$ \\
\hline subcarriers & & $8 \mathrm{k}, 16 \mathrm{k}, 32 \mathrm{k}$ \\
\hline Scattered pilot & $8 \%$ of all & $1 \%, 2 \%, 4 \%$, \\
\hline symbols & & $8 \%$ \\
\hline Continual pilot & $2.6 \%$ of all & $0.35 \%$ \\
\hline symbols & & $8 \%$ \\
\hline \multirow[t]{2}{*}{ Bandwidth } & $6,7,8 \mathrm{MHz}$ & $1.7,5,6,7$ \\
\hline & & $8,10 \mathrm{MHz}$ \\
\hline Typical data & $24 \mathrm{Mbit} / \mathrm{s}$ & $40 \mathrm{Mbit} / \mathrm{s}$ \\
\hline \multicolumn{3}{|l|}{ rates } \\
\hline Max. data rates & 31.7 Mbit/s & $45.5 \mathrm{Mbit} / \mathrm{s}$ \\
\hline \multicolumn{3}{|l|}{ (@20 dB C/N) } \\
\hline Required $\mathrm{C} / \mathrm{N}$ & $16.7 \mathrm{~dB}$ & $10.8 \mathrm{~dB}$ \\
\hline (@24Mbit/s) & & \\
\hline
\end{tabular}

The number of resource blocks is based on the allocated bandwidth. One resource block is defined in the frequency domain as 12 subcarriers for one time slot with duration $0.5 \mathrm{~ms}$. One time slot contains, based on the cyclic prefix choice, 7 or 6 OFDM symbols. The LTE frame is created by 10 subframes with duration $1 \mathrm{~ms}$. Here each subframe contains two time slots. The LTE frame structure can be seen in Fig. 1. 


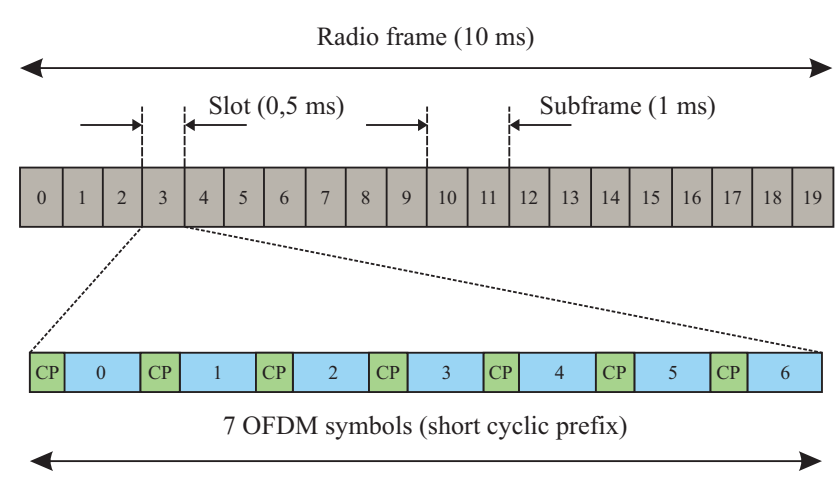

Fig. 1 LTE frame

LTE deploys two different multiple access methods. For uplink SC-FDMA (Single Carrier - Frequency Division Multiple Access) and OFDMA (Orthogonal Frequency Division Multiple Access) for downlink is used. The subcarrier spacing in constant $15 \mathrm{kHz}$ and is not related to the bandwidth. The reason why OFDMA is used at downlink is the higher spectral efficiency, robustness against multi-path fading, scalable bandwidth size, easy MIMO implementation, easy subrarrier coordination etc. Physical Radio Block (PRB) is the basic frequency-time unit in LTE and contains 12 subcarriers placed next to each other for one time slot of $0.5 \mathrm{~ms}$ as it is shown in Fig. 2. It is the smallest resource block assigned by eNode B for frequency planning (180 $\mathrm{kHz} \times 0.5 \mathrm{~ms}$ ). Each PRB is made from resource elements from which everyone represents one subcarrier during one symbol period in OFDM or SC-FDMA. The data communication at downlink can process two code words at the same time and therefore it can use MIMO. LTE at downlink as well as at uplink uses QPSK, 16-QAM and 64-QAM.

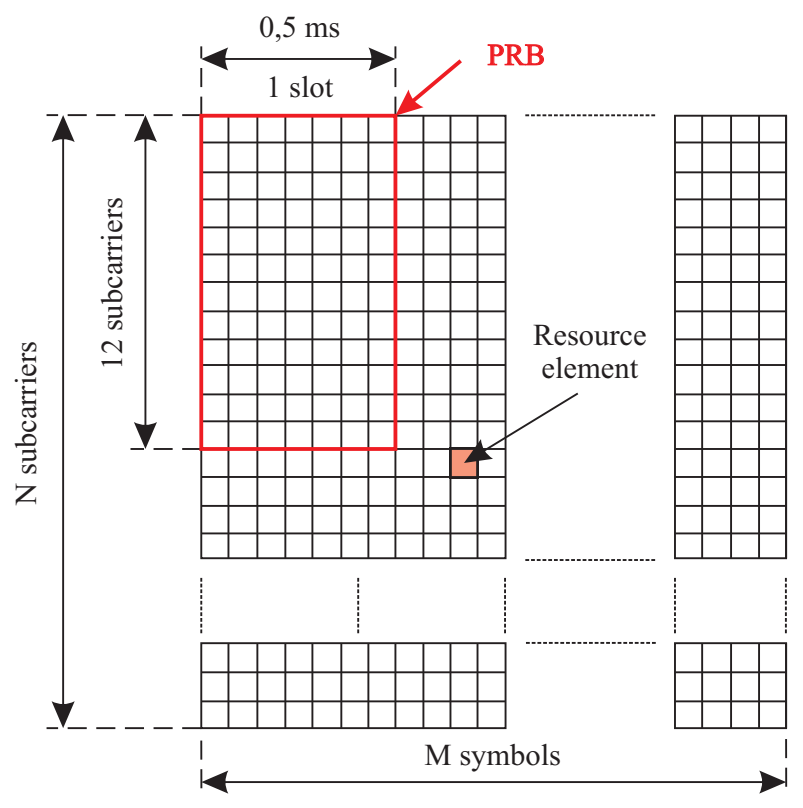

The signal processing at downlink is illustrated in Fig. 3 The data signal is first handled by the channel encoder (FCE), than interleaved and mapped to m-bit symbol based on the used digital modulation technique (QPSK, 16-QAM or 64QAM). Modulated symbols are serial-to-parallel converted and IFFT (Inverse Fast Fourier Transformation) is applied. Every OFDM symbol contains $\mathrm{N}$ samples (each sample contain m-bits). The result of the transformation contains again $\mathrm{N}$ samples, now in the time domain. After the parallel-to-serial conversion the Cyclic Prefix (CP) is inserted. The CP suppress the Inter Symbol Interference (IS) and Inter Carrier Interference (ICI) as well. The transmission signal with the $\mathrm{CP}$ is than converted in the digital-toanalog converter, modulated to the band pass, amplified and transmitted. Detailed LTE parameters are listed in Tab. 2

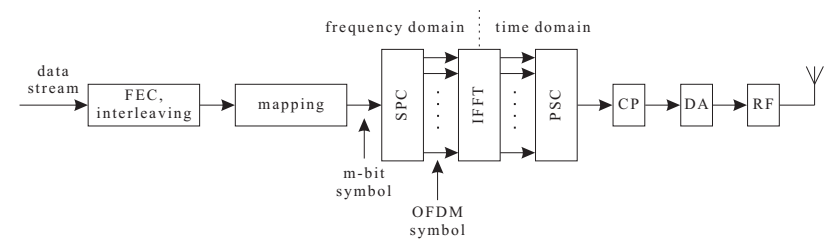

Fig. 3 LTE downlink signal processing

Table 2 LTE parameters

\begin{tabular}{lc}
\hline \hline Parameter & Value \\
\hline \hline Downlink peek values & 100(SISO), 172.8(2x2MIMO), \\
with 64-QAM (Mbit/s) & $326.4(4 \times 4 \mathrm{MIMO})$ \\
\hline Uplink peek values & $50(\mathrm{QPSK}), 57.6(16-\mathrm{Q} A M)$, \\
with single antenna & $86.4(64-\mathrm{QAM})$ \\
\hline Bandwidth $(\mathrm{MHz})$ & $1.4,3,5,10,20$ \\
\hline
\end{tabular}

Delay

From idle to active state:

$$
<100 \mathrm{~ms}
$$

Small packets:

$$
\sim 100 \mathrm{~ms}
$$

\begin{tabular}{lc} 
Modulation & QPSK, 16-QAM, 64-QA \\
& (for uplink and downlink) \\
\hline Multiple access & Downlink: OFDMA \\
methods & Uplink: SC-FDMA
\end{tabular}

Fig. 2 Physical resource block structure 


\section{COEXISTENCE OF DVB-T/T2 AND LTE800}

The demand for modern, effective and intelligent wireless communication systems, which enable the delivery of multimedia services in high quality with constant QoS (Quality of Service) level, is increasing rapidly on the user side as well as provider side. The access whomever, wherever and whenever is the main goal of every mobile and broadcast system in recent times. For that reason, the research on future generation wireless communication is aimed towards the development of effective and robust transmission systems, which can work in large range of the frequency spectrum. To meet these requirements, on the ITU conference WRC-2007 (World Radio Conference) a decision was made, that the frequency spectrum 790-862 $\mathrm{MHz}$ will be allocated to mobile services. On the other hand, this decision, from the point of technical and market view, led to the creation of new interference scenarios and coexistence between two service types that use the same or neighbouring frequency bands. These services are LTE and DVB-T/T2. The implementation of mobile services in the 790-862 MHz band can cause signal interference on the TV receivers or set-up boxes in home environments. The radio interference between various services are generally mitigated by employing guard bands or by ensuring satisfactory geographical separation. For mobile LTE networks, deployed in the UHF band, the guard band was set only to $1 \mathrm{MHz}$ from the edge of CH60 for television broadcasting. In Fig. 4 shows how the frequency band for LTE utilize the spectrum resources $(2 \times 30 \mathrm{MHz}$ for uplink and downlink). The frequency plan, created by CEPT SE42 Group, describes the mode for LTE with $5 \mathrm{MHz}$ and $10 \mathrm{MHz}$ bands arranged in pairs. Reverse duplex system was designed for the base station in lower frequency band and higher frequency band for the uplink.

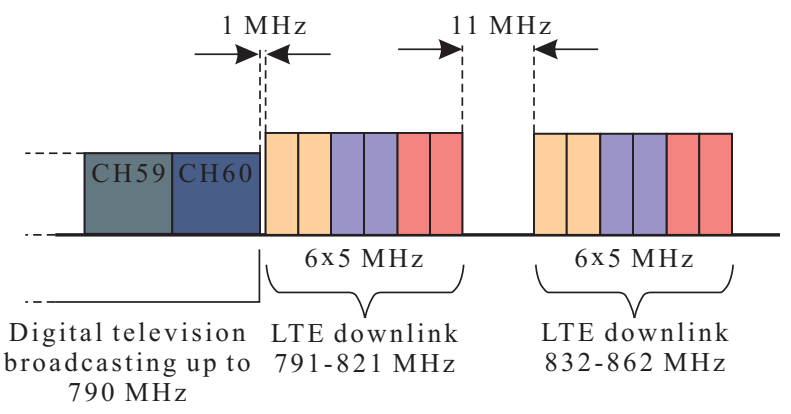

Fig. 4 Frequency spectrum allocation

The close proximity of the transmission frequencies of the LTE base stations and DVB-T transmitters can lead to digital television service interference in some locations, but mainly on the coverage borders. Transmitter power, base station location, mobile services network infrastructure, antennas and network topologies are totally different for digital terrestrial television transmission DVB-T and for cellular mobile networks. Because of these differences and small guard band one can expect that the amount of interferences will raise. Antennas used for reception of television broadcasting installed before the year 2013 were designed to receive VHF Band III and UHF Bands IV and V up to $862 \mathrm{MHz}$, what exceeds Ch60. Since LTE is deployed in the band from $791 \mathrm{MHZ}$ to $862 \mathrm{MHz}$, television systems are subjected by interference, in particular upper channels up to Ch60. The interference can also occur at downlink (from base station to user terminal) as well as uplink (from user terminal to base station). Interference scenarios can be described as follows:

- Digital television influence on mobile systems - in this coexistence scenarios the digital television transmitter can act like the interferer to LTE. Depending on the frequency under usage, LTE base station receiver or LTE user terminal receiver can be influenced.

- LTE influence on digital television - LTE inuence on digital television - in this case, LTE transmitter is the interferer to the digital television receiving equipment. The interference can be caused either by LTE terminal (user equipment) transmitting the up-link signal or LTE base station transmitting the down- link signal. In the case of terrestrial TV signal, neighbouring channel scenarios can occur.

\section{SIMULATION SCENARIO}

For the simulation we employed the ITU-R P.15464 land propagation model. This model is used for environments in which the real world transmitting antenna is placed, i.e. on the hill named Blatný vrch. The distance between the transmitter (Blatný vrch) and receiver (Lučenec) is approximately $8,5 \mathrm{~km}$. This simulation scenario is visualized in Fig. 5. We created the simulation model in SEAMCAT (Spectrum Engineering Advanced Monte Carlo Analysis Tool) [5]. It is a free of charge integrated software tool based on the Monte-Carlo simulation method.

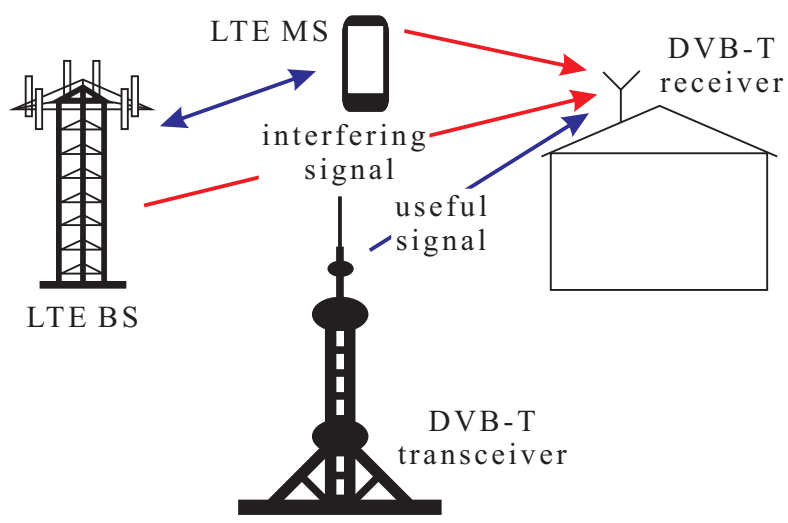

Fig. 5 Interference scenario between DVB-T and LTE

For the sake of clarity we prepared a set of tables, which are shown in the following part, to put together all the simulation parameter for the DVB-T transmitter and receiver as well as the LTE BS. The simulation scenario parameters set-up for the DVB-T transmitter and receiver can be found in Tab. 3 and 4, respectively. Finally, tab. 5 contains parameters of the LTE system. 
Table 3 DVB-T receiver parameters

\begin{tabular}{lc}
\hline \hline Parameter & Value \\
\hline \hline Antenna hight & $10 \mathrm{~m}$ \\
\hline Antenna radiation diagram & DVB-T ITU-R BT.419 \\
\hline Antenna gain & $16 \mathrm{dBi}$ \\
\hline Channel bandwidth & $8 \mathrm{MHz}$ \\
\hline \hline
\end{tabular}

text

Table 4 DVBT-T transmitter parameters

\begin{tabular}{lc}
\hline \hline Parameter & Value \\
\hline \hline Mast height & $10 \mathrm{~m}$ \\
\hline Transmission frequency & $786 \mathrm{MHz}(\mathrm{Channel} \mathrm{60)}$ \\
\hline Antenna gain & $0 \mathrm{dBi}$ \\
\hline Polarization & Vertical \\
\hline ERP & $15.85 \mathrm{~kW}$ \\
\hline \hline
\end{tabular}

text

Table 5 LTE system parameters

\begin{tabular}{lc}
\hline \hline Parameter & Value \\
\hline \hline System & OFDMA DL \\
\hline Transmission frequency & $794 \mathrm{MHz}$ \\
\hline Bandwidth & $10 \mathrm{MHz}$ \\
\hline BS transmission power & $46 \mathrm{dBm}$ \\
\hline BS antenna hight & $30 \mathrm{~m}$ \\
\hline BS antenna gain & $15 \mathrm{dBi}$ \\
\hline Users per BS & 20 \\
\hline Number of BS & 7 \\
\hline Cell radius & $2 \mathrm{~km}$ \\
\hline UE antenna hight & $1.5 \mathrm{~m}$ \\
\hline UE antenna gain & $0 \mathrm{dBi}$ \\
\hline Propagation model & Extended Hata \\
\hline \hline
\end{tabular}

\section{RESULTS}

\subsection{Simulation scenario \#1}

The analysed scenario involves the LTE mobile radio system and DVT-T transmitter as well as receiver. In this case we consider interference which is generated by a certain number of LTE base stations. These base stations disturb the digital television reception. We would like to mention the second multiplex on channel 60 in the surrounding of the town Lučenec (Slovakia) as an example. In simulation scenario \#1 no filter at the DTV receiver nor SEM at the LTE base station was used. The results are summarized in Tab. 6. The final value of the interference signal is $-32.06 \mathrm{dBm}$, whereas the desired signal strength is -46.01 $\mathrm{dBm}$. Therefore, we can see that the value of the interference signal strength is higher than the desired signal. This gives us a significant assumption for interference occurrence, since the designated C/I (Carrier-to-Interference) ratio is not fulfilled. After the simulation, we inserted the results in the so called interference calculator, in which the high probability of interference, i.e. $100 \%$, is confirmed. Fig. 6 compares the dRSS and iRSS values of the LTE and DVB-T signals.

Table 6 Simulation results for simulation scenario \#1

\begin{tabular}{lc}
\hline \hline Parameter & Value \\
\hline \hline dRSS mean value & $-46.1 \mathrm{dBm}$ \\
\hline iRSS mean value & $-32.06 \mathrm{dBm}$ \\
\hline $\mathrm{C} / \mathrm{I}$ & $-13.95 \mathrm{~dB}$ \\
\hline Probability of interference & $100 \%$ \\
\hline \hline
\end{tabular}

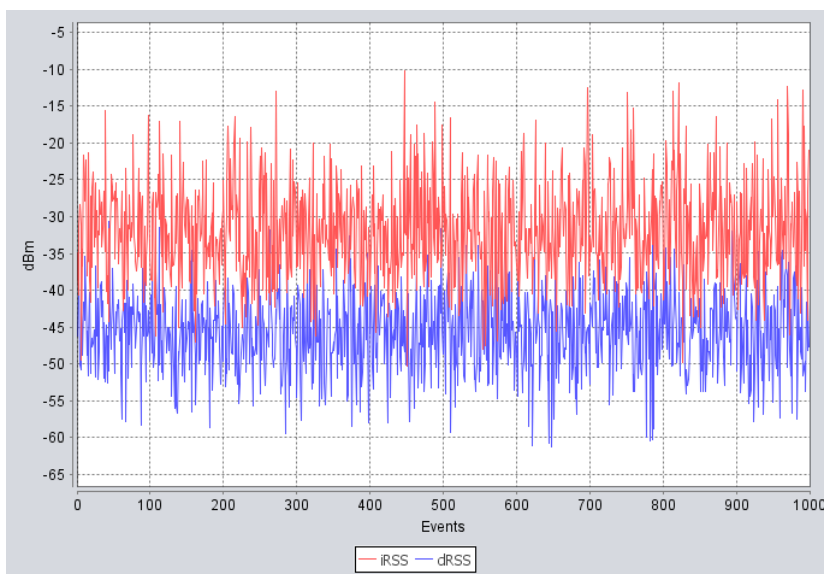

Fig. 6 dRSS and iRSS values 


\subsection{Simulation scenario \#2}

In scenario \#2, a spectral emission mask (SEM) was used on the LTE transmitter. The SEM on the LTE base station is designed according to CEPT Report 30 [6]. The interference signal has dropped down to $-66.45 \mathrm{dBm}$. The received signal strength is almost same as in the first scenario. The ration between the received and interference signal is $20.49 \mathrm{~dB}$. The $\mathrm{C} / \mathrm{I}$ values is however not satisfying since it is $24 \mathrm{~dB}$ and hence it does not meet the required values. On the other hand we assumed that the interference will be smaller and indeed the calculator showed a $49.9 \%$ probability of interference. Tab. contains the simulation results for scenario \#2 with the SEM on the LTE base station. The values of dRSS and iRSS are shown in Fig. 7

Table 7 Simulation results for simulation scenario \#2

\begin{tabular}{lc}
\hline \hline Parameter & Value \\
\hline \hline dRSS mean value & $-45.96 \mathrm{dBm}$ \\
\hline iRSS mean value & $-66.45 \mathrm{dBm}$ \\
\hline $\mathrm{C} / \mathrm{I}$ & $20.49 \mathrm{~dB}$ \\
\hline Probability of interference & $49.9 \%$ \\
\hline \hline
\end{tabular}

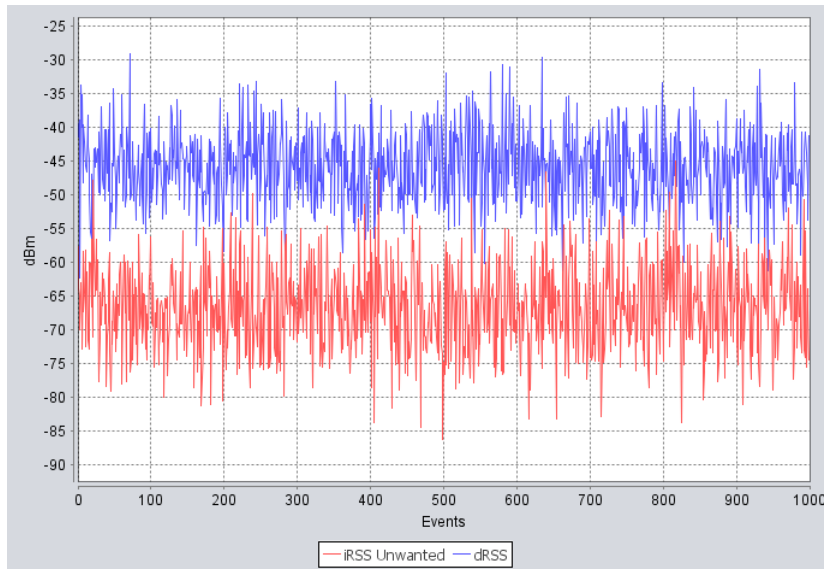

Fig. 7 dRSS and iRSS values

\subsection{Simulation scenario \#3}

In this scenario we applied the filter Mugler NFDD787 at the input of DVB-T receiver to suppress the LTE interference. The final value of the interference signal has significantly decreased to $-92.98 \mathrm{dBm}$. The ration between the received and interference signal is $47.04 \mathrm{~dB}$. This values is higher than the limit values of $23.04 \mathrm{~dB}$ and therefore the requirements are met. The interference value in scenario \#3 is $0.3 \%$ which can be considered as negligible. The simulation results are pointed out in Tab. 8. The comparison of dRSS and iRSSvalues are shown in Fig. 8. From the comparison of each scenario one can conclude that application the appropriate suppression filter seams to be the best solution to mitigate the impacts of interference.
Table 8 Simulation results for simulation scenario \#3

\begin{tabular}{lc}
\hline \hline Parameter & Value \\
\hline \hline dRSS mean value & $-45.94 \mathrm{dBm}$ \\
\hline iRSS mean value & $-92.98 \mathrm{dBm}$ \\
\hline C/I & $47.04 \mathrm{~dB}$ \\
\hline Probability of interference & $0.3 \%$ \\
\hline \hline
\end{tabular}

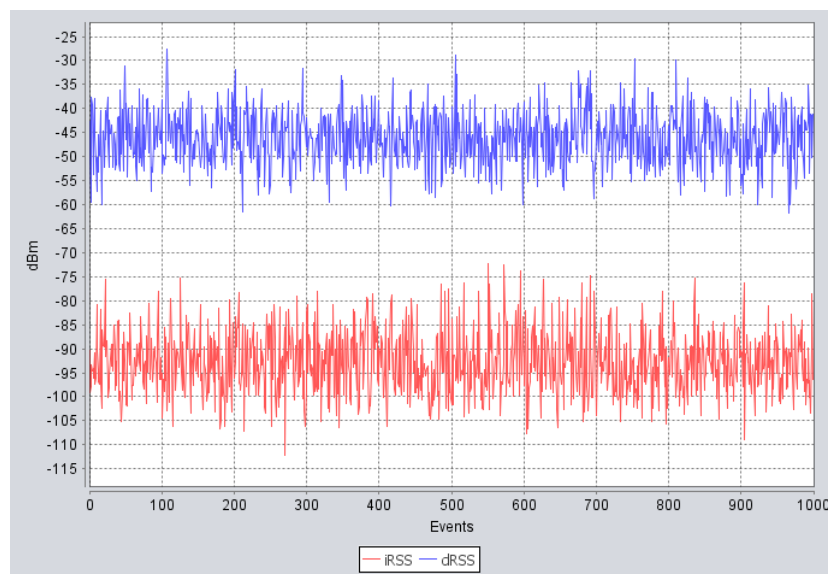

Fig. 8 dRSS and iRSS values

\section{CONCLUSIONS}

The aim of this paper was to analyse the problematic of interference between LTE mobile systems and DVB$\mathrm{T}$ television broadcast systems. Since the LTE system is under deployment and in some parts of Slovakia already in operation, it is very meaningful to investigate the impact of the coexistence between these technologies, hence it affects a large part of the population. To estimate the effects of the interaction of the above mentioned systems and calculate the particular values of the signal parameters we used SEAMCAT as a simulation tool. The simulations have shown that the best solution to mitigate the interference were reached in the case when filter war used on the DVB-T receiver input. We can therefore conclude that the best protection of the DVB-T reception influenced by LTE transmission is the application of a suitable filter, which can effectively suppress the interference signal. These simulation results have also an other meaning, whereas these were used as a base line to the real world measurements which were done in the area of the town Lučnec. All these simulations and measurements lead to the creation of of an interactive map (reachable at maps.fei.tuke.sk) where the wide population can find the infer fence map for the Slovakia area. This paper as well as the real world measurements and the interactive map are results of a tight cooperation between academia (Technical university of Košce), The Research Institute of Posts and Telecommunications and industry (Alcatel-Lucent). 


\section{ACKNOWLEDGEMENT}

This work was supported by the Slovak Research and Development Agency under the contract No. APVV-0696-12.

\section{REFERENCES}

[1] ITU,"Final Acts - WRC-07," WORLD RADIOCOMMUNICATION CONFERENCE (GENEVA, 2007), 2007.

[2] EBU Tech, "Frequency and Network Planning Aspects of DVB-T2," in ETSI EN 300744 V1.6.1 (2009. 01) European Standard (Telecommunications series), 2009.

[3] ETSI, "Digital Video Broadcasting (DVB); Framing structure, channel coding and modulation for digital terrestrial television," in EBU Tech 3348, Geneva, 2012.

[4] ETSI, "Digital Video Broadcasting (DVB); Framing structure, channel coding and modulation for a second generation digital terrestrial television broadcasting system (DVB-T2)," in ETSI EN 302755 V1.4.1 (2015-07) European Standard (Telecommunications series), 2015.

[5] ECO, "SEAMCAT Handbook,", January 2010. SEAMCAT Handbook

[6] CEPT Report 30 , "The identification of common and minimal (least restrictive) technical conditions for 790 - $862 \mathrm{MHz}$ for the digital dividend in the European Union,", October 2009.

Received January 4, 2016, accepted January 21, 2016

\section{BIOGRAPHY}

Lukáš Sendrei was born on 1. 11. 1987. In 2012 he graduated (M.Sc.) with honours at the Department of Electronic and Multimedia Telecommunication of the Faculty of Electrical Engineering and Informatics at Technical University in Košice and currently he is working towards his $\mathrm{PhD}$ at the same university. In 2013, he spend two months with the Vodafone Chair Mobile Communications Systems, Technische Universität Dresden, Dresden, Germany as a guest researcher working in the area of nonlinear effects in GFDM. His current research interests include wireless multicarrier communication systems (FBMC, GFDM) for $5 \mathrm{G}$ and cognitive radio systems.
Marián Felix was born on 9 July 1971. He studied at the Faculty of Electrical Engineering, Department of Radio Technology at Slovak University of Technology in Bratislava where he graduated in 1994. After short practice in the JGT theater sound studio in Zvolen in 1995, he worked as engineer and designer in the department of development, construction and projection at Aircraft service station in Banská Bystrica. In this work he was engaged in several development projects for radio equipment intended primarily for military aviation sector. Since 2007 until now he works in the Division of Radiocommunication Services of the Research Institute of Posts and Telecommunications in Banská Bystrica. Currently he is the director of this division. He participated in a number of research projects as the principal investigator and as a member of the research team. He is specialized in the area of digital broadcast radio and television, especially in the area of system and technology. Many of his projects have found practical applications.

Juraj Oravec graduated at the Faculty of Electrical Engineering of Czech Technical University in Prague in the field of Telecommunications, specialization Video Frequency Technique in 1975. From 1977 he has been working in the Research Institute of Posts and Telecommunications Prague (VS) in Banská Bystrica. Currently he holds the position of Deputy Director General for Radiocommunications. During his career in VÚS he was specialized on the issues of processing and distribution of TV and radio signals through CATV/MATV systems and terrestrial digital broadcasting, as well as the issues of frequency planning and frequency management.

Stanislavav Marchevský received MSc in electrical engineering at the Faculty of Electrical Engineering, Czech Technical University in Prague, in 1976 and $\mathrm{PhD}$ degree in radioelectronics at the Technical University of Košice in 1985. Currently he is a Professor of Electronics and Multimedia Communications Department of Faculty of Electrical Engineering and Informatics of Technical University of Košice. He teaching interests include switching theory, digital television technology, and satellite communications. His research interests include image nonlinear filtering, neural networks, genetic algorithms, and multiuser detection, space-time communication, diversity communication over fading channel, and power and bandwidth efficient multiuser communications. 